\title{
El método de la transferencia de beneficios para la valoración económica de bienes de no mercado generados por los eventos deportivos
}

\section{Joan Mogas Amoros'}

\section{Universidad Rovira i Virgili} Email: joan.mogas@urv.cat

RESUMEN: La práctica de un deporte o la celebración de un evento deportivo generan un conjunto de flujos económicos que es preciso medir. Sin embargo, la evaluación del impacto económico es una tarea difícil, no sólo por la dificultad de la obtención de los datos, sino también por las diferentes metodologías que pueden ser utilizadas para elaborar magnitudes económicas del deporte. Una de las metodologías es el análisis coste-beneficio (ACB) que permite evaluar proyectos o eventos desde una óptica pública, y que implica evaluar todos los costes y todos los beneficios, tanto privados como sociales. El objetivo de este estudio es presentar la transferencia de beneficios para la valoración de los impactos de bienes púbicos asociados a eventos o infraestructuras deportivas, y que es necesaria para poder realizar el ACB. La transferencia de beneficios, que consiste en la aplicación de los resultados de estudios de valoración monetaria a nuevos contextos de política, puede resultar una alternativa muy atractiva respecto a hacer una nueva investigación que implica tiempo y dinero, permitiendo así acelerar la toma de decisiones.

PALABRAS CLAVE: Impacto económico de eventos deportivos, análisis costebeneficio, bienes públicos, transferencia de beneficios.

\section{The benefit transfer method for the economic valuation of the Non-Market value of Sports Events}

\begin{abstract}
The practice of a sport or the celebration of a sporting event generates a set of economic flows that must be measured. However, the evaluation of the economic impact is a difficult task, not only because of the difficulty in obtaining the data, but also because of the different methodologies that can be used to estimate the economic magnitudes of the sport. One of the methodologies is the cost-benefit analysis (CBA) that allows evaluating projects or events from a public perspective. The CBA involves evaluating all costs and all benefits, both private and social. The objective of this paper is to present the benefit transfer method, in the estimation of the impacts of public goods associated with sports events or infrastructures, and that is necessary to carry out the ACB. The benefit transfer, a technique in which the results of studies on monetary valuations are applied to new policy contexts, can result as an attractive alternative respect to conducting a new research that implies time and money, thus allowing faster decision making.
\end{abstract}

KEY WORDS: Economic impact of sporting events, cost-benefit analysis, public goods, benefit transfer. 


\section{INTRODUCCIÓN}

La práctica de un deporte o la celebración de un evento deportivo generan un conjunto de flujos económicos que es preciso medir. La evaluación del impacto económico de un evento deportivo es una tarea difícil, no sólo por la dificultad de la obtención de los datos, sino también por las diferentes metodologías que pueden ser utilizadas para elaborar magnitudes económicas del deporte.

La investigación sobre impacto económico del deporte presenta un importante crecimiento a partir del año 2000 (Salgado-Barandela et al., 2017). En Europa, las primeras investigaciones sobre la economía del deporte las encontramos en Francia en los años 70, aunque no sería hasta el 1988 cuando se iniciaron los primeros intentos rigurosos de medir la incidencia económica del deporte en sus estados miembros, a instancias del Comité Directivo para el Desarrollo del Deporte del Consejo de Europa (CDSS) (Jones, 1989; Andreff et al., 1995). En España los primeros estudios sobre impacto económico no se llevan a cabo hasta principios de los años 90 (Pedrosa y Salvador, 2003).

Los métodos que se han venido utilizado tradicionalmente para la medición de la incidencia económica del deporte son: las Cuentas Satélite, las Tablas Input-Output (TIO), el modelo de Equilibrio General, el Análisis Coste Beneficio (ACB), la Valoración Contingente, así como los análisis sectoriales y regionales. Los tres primeros presentan un enfoque macroeconómico mientras los tres últimos presentan un enfoque parcial de cuentas, o de indicadores del deporte, con una dimensión infranacional (véase Pedrosa y Salvador (2003) y Barajas et al. (2012), para una explicación detallada de cada uno de los métodos de valoración). Las metodologías existentes no son excluyentes entre sí, por el contrario, se complementan. Sin embargo los estudios realizados muestran falta de unanimidad con respecto al método más adecuado y sus limitaciones, convirtiendo la valoración de los eventos deportivos en una labor compleja (Pedrosa y Salvador 2003; Salgado-Barandela et al., 2017).

De los diferentes métodos, el Análisis Coste-Beneficio (ACB) es un método amplio y flexible, que permite identificar y evaluar las repercusiones socioeconómicas de proyectos y programas públicos o eventos deportivos, al objeto de determinar su (no) justificación desde un punto de vista económico. Para ello requiere evaluar todos los costes y beneficios, tanto privados como sociales, que se pueden derivar de la implantación de cada proyecto. Exige pues el ACB la internalización de todos los efectos y su monetización, de un modo especial las externalidades o bienes públicos que afectan al bienestar de la población pero no se transaccionan en los mercados (LópezCasasnovas y Vegara, 2013).

El objetivo de este estudio es presentar la transferencia de beneficios como una alternativa muy atractiva en la valoración de los impactos de bienes púbicos asociados a eventos o infraestructuras deportivas, y que es necesaria para poder realizar el ACB, con respecto a hacer una nueva investigación que implica tiempo y dinero, permitiendo acelerar la toma de decisiones. 


\section{EL ACB Y LA TRANSFERENCIA DE BENEFICIOS}

El deporte representa en la actualidad una de las actividades más destacadas de cualquier sociedad desarrollada, con un impacto tanto social como económico. Por todo ello es importante valorar la importancia económica y social cuando una ciudad o país organiza un evento deportivo, y más cuando muchas veces una parte del coste de estos eventos es sufragado por las administraciones, ya sea a través del ofrecimiento de facilidades administrativas o de infraestructuras 0 , directamente, mediante subvenciones o patronazgo.

Una técnica adecuada para la evaluación de políticas de gasto público es el ACB. El ACB necesita incorporar todos los costos y todos los beneficios y su monetización a fin de determinar si hay algún beneficio neto. El ACB permite la comparación entre diferentes alternativas $\mathrm{y}$, a partir del cálculo de diferentes medidas, como el valor actual neto, la ratio coste-beneficio o la tasa interna de retorno, permite elegir qué alternativa proporcionan mayor rentabilidad desde el punto de vista social (Ortega, 2012). El ACB desarrolla un marco metodológico que costa de diferentes etapas. Éstas van desde la identificación del proyecto, la selección de todos los costes y los beneficios, su expresión en términos monetarios, el cálculo de los diferentes indicadores de rentabilidad y el análisis de sensibilidad (European Commission, 2008; De Rus, 2010; Dasgupta y Pearce, 1972).

EI ACB puede ser muy útil para evaluar impactos socioeconómicos derivados de la celebración de eventos deportivos, siendo una importante herramienta para la toma de decisiones por parte de los organizadores (Hurtado et al. 2007, Késenne, 2005). Si bien es el enfoque más adecuado para valorar el impacto sobre el bienestar, hay pocos estudios en los que se aplique el ACB para evaluar eventos deportivos (Schaffer et al., 2003; Mules y Dwyer, 2005; Taks et al., 2011; De Nooij et al., 2013) y menos en España (Hurtado et al., 2007; Barajas y Sánchez, 2011; Jiménez-Naranjo et al., 2016).

Una de las dificultades del ACB, es que realizarlo en toda regla es una misión compleja, ya que requiere una gran cantidad de datos específicos que a menudo faltan y son muy difíciles de recopilar. En concreto, muchas de las externalidades de las actuaciones públicas, tanto en beneficios como en costes. La valoración monetaria de los impactos no internalizados por los mercados y, como consecuencia no tienen precio, es un elemento clave en el ACB (López-Casasnovas y Vegara, 2013). Por ejemplo, efectos externos asociados a un evento deportivo pueden ser efectos positivos, como la mejora de la imagen del municipio, la mejora del bienestar de la población en términos de un aumento de la participación deportiva y un estilo de vida más saludable. $O$ negativos, como un incremento en la congestión del tráfico o del ruido debido al evento deportivo.

Para hacer frente a la evaluación en términos monetarios de los bienes y servicios de no mercado se ha desarrollado un conjunto de procedimientos para evaluar las externalidades. Dentro de estos métodos se encuentran métodos de preferencias declaradas, como el coste de viaje o precios hedónicos, que realizan la evaluación analizando los comportamientos observados y los de preferencias declaradas, con el método de la valoración contingente, que se basa en el análisis a reacciones a cambios potencialmente planteados mediante técnicas de encuesta. Sin embargo, muchas veces la implementación de estas metodologías pueden comportar unos altos costos de implementación, tanto económicos como en el tiempo requerido (Azqueta, 1994).

Cuando el costo es un factor restrictivo, se puede recurrir a técnicas como la transferencia de beneficios y el meta-análisis (Brouwer, 2000). Estas técnicas se basan en fuentes de datos secundarias e implican la medición de la disposición a pagar 
utilizando estimaciones de eventos relacionados. En concreto, y tal como lo definen Rosenberger y Loomis (2003), "la transferencia del beneficios es la adaptación de la información derivada desde una investigación original para la aplicación en un contexto diferente de estudio". El sitio donde se ha realizado el estudio se conoce normalmente como sitio de estudio, y el sitio de interés de la política se conoce como sitio de política.

Existen tres vías por las que se realiza la transferencia de beneficios: la transferencia del valor unitario, que consiste en la transferencia de un valor procedente de un estudio primario preexistente a un nuevo estudio; la transferencia de beneficios de funciones, que consiste en la estimación de una función a partir de un único estudio primario o la transferencia de funciones de meta-análisis, donde funciones de diferentes estudios se combinan en una función de transferencia de beneficios para el sitio de política (Rosenberger y Loomis, 2003).

La transferencia de beneficios comporta riesgos importantes de obtener resultados sesgados. Este caso se puede dar, por ejemplo, cuando el bien ya estudiado difiere notablemente del bien al que se quieren extrapolar las estimaciones, tanto en los impactos físicos como en la población afectada. En los últimos años se han propuesto aproximaciones más rigurosas a la transferencia de beneficios, como el uso de diversos protocolos que tienen en cuenta los factores que pueden afectar significativamente a los valores estimados (Boyle y Bergstrom, 1992; Rosenberger y Loomis, 2003; Johnston et al., 2015). Una limitación de estos protocolos es que es muy difícil encontrar en la práctica aplicaciones que permitan cumplir todas las recomendaciones sugeridas. Por todo ello sigue abierto el campo de investigación sobre la validez de las estimaciones basadas en la transferencia de beneficios.

Aunque en la mayoría de los casos se prefiere el uso de investigación primaria de alta calidad para estimar valores, las realidades del proceso de toma de decisiones, en particular, limitaciones de tiempo y presupuesto, a menudo determinan que la transferencia de beneficios es la única opción viable que nos da la información requerida. Dadas estas realidades, la transferencia de beneficios se ha convertido en un componente central de prácticamente todos los análisis de costo-beneficio a gran escala (Smith et al., 2002). Por lo tanto, aunque las transferencias de beneficios están sujetas a una variedad de posibles errores y es una técnica controvertida, la literatura reconoce cada vez más la necesidad de la información resultante (Johnston y Rosenberger, 2010).

\section{LA TRANSFERENCIA DE BENEFICIOS EN LA VALORACIÓN DE BIENES PÚBLICOS GENERADOS POR EVENTOS DEPORTIVOS}

La transferencia de beneficios es una práctica habitual en campos como la economía del transporte, de la salud o de la economía ambiental donde los análisis coste-beneficio utilizan con frecuencia valores del tiempo, de accidentes, de la vida humana o de bienes y servicios ambientales, transferidos de algún otro estudio. En el campo de la economía ambiental se ha aplicado desde la gestión de la calidad del agua, la gestión de residuos o a la gestión forestal (Brouwer, 2000).

Sin embargo, ésta no es todavía una forma habitual de proceder en la economía del deporte. Sólo encontramos una aplicación de transferencia del valor unitario de la disposición a pagar en Taks et al. (2011) y De Nooij (2014). Taks et al. (2011) argumentan que el ACB ofrece una imagen precisa y realista de los costes y beneficios reales de la organización de un evento deportivo. En dichos estudios se realiza un ACB 
de acoger un evento deportivo medio como fue el Campeonato Panamericano de Atletismo Junior celebrados en Windsor (Canadá) el 2005. En el ACB, para estimar el valor de los bienes públicos generados por el campeonato se utiliza la disposición a pagar media por la construcción de dos estadios en Lexington (EEUU) obtenidos por Johnson y Whitehead (2000) multiplicado por el número de hogares de Windsor el 2005. Sin embargo, en dichos trabajos, la transferencia de beneficios no es fiable, al no realizarse ningún test de fiabilidad y validez de los resultados, como comporta todo ejercicio de transferencia de beneficios.

Una de las posibles causas de su no aplicación en la valoración económica de eventos deportivos puede ser, por una parte por la complejidad de algunos de los bienes públicos generados por un evento deportivo, como por ejemplo externalidades positivas como la cohesión, la paz social o el orgullo de ciudad o externalidades negativas como el gamberrismo o el dopaje, y que pueden aumentar o disminuir el valor del evento. Además, la obtención de los valores de bienes públicos a través de los métodos de valoración de bienes de no mercado también presenta una serie problemas metodológicos y limitaciones que dificultan la obtención de estos valores. Sin embargo la importante y creciente investigación en los últimos años en valoración de bienes de no mercado puede facilitar el futuro de la transferencia de beneficios.

Por otra parte, otra posible razón puede ser la falta de un protocolo unificado para guiar el diseño y la evaluación de las pautas de transferencia de beneficios, como se ha realizado en otros campos de la economía. Además no existen estudios primarios en el campo de la economía del deporte donde se hayan aplicado métodos de valoración y estimado el mismo tipo de bien que se está considerando para el sitio de la política.

\section{CONCLUSIONES}

El $\mathrm{ACB}$, constituye una importante metodología para la toma de decisiones por parte de los organizadores de eventos deportivos. Esta metodología requiere evaluar todos los costes y los beneficios, tanto privados como sociales, que pueden derivar del evento. De un modo especial, ello requiere evaluar las externalidades ignoradas por los mercados existentes, y presentes en los eventos deportivos. Por ello, las instituciones gubernamentales y las organizaciones privadas tienen con frecuencia la necesidad de estimar los valores de los bienes y servicios de no mercado asociados a un evento deportivo con el fin de realizar un a ACB. Sin embargo, en muchas situaciones, no es práctico o posible obtener la información sobre la cual basar las estimaciones de valoración económica.

La transferencia de beneficios, basada en la aplicación de los resultados obtenidos en otros estudios, es una opción alternativa atractiva con respecto a la realización de una nueva investigación, al requerir generalmente menos tiempo y recursos. Cuando los estudios primaros no están disponibles, son considerados inviables o demasiado caros, sin la transferencia de beneficios el valor de muchos bienes o servicios de no mercado no serían reconocidos o, al menos valorados, en los procesos de toma de decisiones. La transferencia de beneficios puede ser la solución second best cuando hay restricciones de tiempo, financiación, métodos analíticos o los datos impiden el uso de los estudios primarios para proporcionar la información necesaria. La transferencia de beneficios está sujeta a una serie de errores potenciales, que pueden llevar a obtener resultados sesgados, por lo que su aplicación requiere mucha atención tanto en la aplicación del método como de los datos usados. 
A pesar de su uso cada vez más extendido, no hay constancia de su aplicación correcta en los ACB para el análisis del impacto socioeconómico de los eventos deportivos. Por todo ello, hay una reconocida necesidad de una mayor investigación para asegurar una mayor comprensión de la transferencia de beneficios en la valoración del impacto económico y social de eventos deportivos. Un aspecto importe sería elaborar un marco conceptual unificado para guiar el diseño y la evaluación de las pautas para hacer más fiable las aplicaciones de la transferencia de beneficios en un ACB para la medición del impacto económico del deporte y construir una base de datos. Esta guía debería incluir tanto las fortalezas como las limitaciones de los métodos y datos utilizados, las suposiciones realizadas, así como la interpretación de las estimaciones de los beneficios obtenidos. Una de las maneras de abordarlo sería a través del uso del creciente número de expertos y publicaciones sobre cómo utilizar la transferencia de beneficios.

De esta manera, a través de una cuidadosa atención al método de la transferencia de beneficios, los investigadores podrán aprovechar de manera óptima este cuerpo de trabajo para proporcionar la orientación política más precisa y útil en la toma de decisiones acerca de los costes y beneficios de una determinada política o evento deportivo de forma igualmente rigurosa y más coste-eficiente.

\section{REFERENCIAS}

Andreff, W., Bourg, J. R; Halba, B. y Nys, J.F. (1995).Les enjeux econòmiques du sport en Europe: financement et impact économique. París: Dalloz.

Azqueta, Oyarzun, D. (1994). Valoración Económica de Impactos Ambientales. Madrid: McGraw Hill.

Barajas, A. y Sánchez, P. (2011). Aplicación del análisis coste-beneficio (ACB) al Campeonato de España de Natación Master 2011. I Gijón Workshop de Economía del Deporte: El Impacto Económico en el Deporte, Gijón, Universidad de Oviedo.

Barajas, A., Salgado, J. y Sánchez, P. (2012). Problemática de los estudios de impacto económico de eventos deportivos. Estudios de economía aplicada 30 (2), 441462.

Boyle, K.J. y Bergstrom, J.C. (1992). Benefit transfer studies: myths, pragmatism and idealism. Water Resources Research, 28(3), 657-663.

Brouwer, R. (2000). Environmental value transfer: state of the art and future prospects. Ecological Economics, 32(1), 137-152.

Dasgupta, A. K., y Pearce, D W. (1972). Cost-Benefit Analysis: Theory and Practice. London: Macmillan.

De Nooij, M.R., Van Den Berg, M.R., y Koopmans C.C. (2013). Bread or games. A Social Cost-Benefit Analysis of the World Cup Bid of the Netherlands and the Winning Russian Bid. Journal of Sports Economics, 14(5), 521 - 545.

De Nooij, M.R. (2014). Economic Impact Analysis versus cost Benefit Analysis for a Medium Sized Sport Event, a further Improvement. Recuperado de https://papers.ssrn.com/sol3/papers.cfm?abstract id=2393427 
De Rus, G. (2010). Introduction to cost-benefit analysis: looking for reasonable shortcuts. Cheltenham: Edward Elgar.

European Commission (2008). Guide to Cost-Benefit Analysis of investment Projects. Bruselas, European Commission Regional Policy.

Hurtado, J.M., Ordaz Sanz, J.A., y Rueda Cantuche, J.M. (2007). Evaluación del impacto económico y social de la celebración de grandes eventos deportivos a nivel local: el caso del Campeonato de Tenis femenino de la ITF en Sevilla en 2006. Revista de métodos cuantitativos para la economía y la empresa, 3(1), 20-39.

Jiménez-Naranjo, H.V., Coca-Pérez, J. L., Gutiérrez-Fernández, M., SánchezEscobedo, M.C. (2016). Cost-benefit analysis of sport events: The case of World Paddle Tour. European Research on Management and Business Economics, 22(3), 131-138.

Johnson, B., y Whitehead, J. (2000). Value of public goods from sports stadiums: The CVM approach. Contemporary Economic Policy, 18, 48-58.

Johnston, R.J. y Rosenberger, R.S. (2010). Methods, Trends and Controversies in Contemporary Benefit Transfer. Journal of Economic Surveys, 24 (3), 479-510.

Johnston, R.J., Rolfe, J., Rosenberger, R. y Brouwer, R. (2015). Introduction to Benefit Transfer Methods. En Johnston, R.J., Rolfe, J., Rosenberger, R. y Brouwer, R. (Eds.). Benefit Transfer of Environmental and Resource Values. A Guide for Researchers and Practitioners (pp. 19-59). Dordrecht: Springer.

Jones, H. (1989). The economic impact and importance of sport: a European study. Committee for the Development of Sport. Strasbourg: Council of Europe.

Késenne, S. (2005). Do We Need an Economic Impact Study or a Cost-Benefit Analysis of a Sports Event? European Sport Management Quartely, 5 (2), 133-142.

López-Casasnovas, G., y Vegara Carrió, J. (2013). El análisis coste-beneficio y la toma de decisiones. Presupuesto y gasto público, 69, 107-115.

Mules, T., y Dwyer, L. (2005). Public sector support for sport tourism events: The role of cost benefit analysis. Sport in Society, 8, 338-355.

Ortega, B. (2012). Análisis Coste-Beneficio. eXtoikos, 5, 147-149.

Pedrosa Sanz, R. y Salvador Insúa, J. (2003). El impacto del deporte en la economía. Problemas de medición. Revista Asturiana de Economía, 26, 61-84.

Rosenberger, R.S., y Loomis, J.B. (2003). Benefit Transfer. En Champ, P.A., Boyle, K.J. y Brown T.C. (eds.). A Primer on Nonmarket Valuation (pp. 445-482). Dordrecht: Springer.

Salgado-Barandela, J., Barajas, A. y Sánchez-Fernández, P. (2017). Impacto económico del deporte: tema de interés creciente en la literatura científica. Revista Internacional de Medicina y Ciencias de la Actividad Física y el Deporte, 17(68), 729-755.

Schaffer, M., Greer, A., y Mauboulos, C. (2003). Olympic costs and benefits: A costbenefit analysis of the proposed Vancouver 2010 Winter Olympic and Paralympic Games. Vancouver, Canada: Canadian Centre for Policy Alternative. 
Smith, V. K., Van Houtven, G., y Pattanayak, S. K. (2002). Benefit transfer via preference calibration: "Prudential algebra" for policy, 78 (1), 132-152.

Taks,M., Kesenne,S.,Chalip, L.,Green,B., y Martyn,S. (2011). Economic impact analysis versus cost benefit analysis: The case of a medium-sized sport event. International Journal of Sport Finance, 6, 187-203. 